This document is confidential and is proprietary to the American Chemical Society and its authors. Do not copy or disclose without written permission. If you have received this item in error, notify the sender and delete all copies.

\title{
A New Strategy for Intestinal Drug Delivery via pH- Responsive and Membrane-Active Nanogels
}

\begin{tabular}{|r|l|}
\hline Journal: & ACS Applied Materials \& Interfaces \\
\hline Manuscript ID & am-2018-15661j.R1 \\
\hline Manuscript Type: & Letter \\
\hline Autho Submitted by the & 07-Oct-2018 \\
\hline Complete List of Authors: & $\begin{array}{l}\text { Wang, Shiqi; Imperial College London, Department of Chemical } \\
\text { Engineering } \\
\text { Ha, Youlim; Imperial College London, Department of Chemical } \\
\text { Engineering } \\
\text { Huang, Xiaozhen; Imperial College London, Department of Chemical } \\
\text { Engineering } \\
\text { Chin, Benjamin; Imperial College London, Department of Chemical } \\
\text { Engineering } \\
\text { Sim, Wen; Imperial College London, Department of Chemical } \\
\text { Engineering } \\
\text { Chen, Rongjun; Imperial College London, Department of Chemical } \\
\text { Engineering }\end{array}$ \\
\hline
\end{tabular}




\title{
A New Strategy for Intestinal Drug Delivery via $\mathrm{pH}-$ Responsive and Membrane-Active Nanogels
}

\author{
Shiqi Wang, Youlim Ha, Xiaozhen Huang, Benjamin Chin, Wen Sim, \\ and Rongjun Chen* \\ Department of Chemical Engineering, Imperial College London, \\ South Kensington Campus, London SW7 2AZ, UK
}

\begin{abstract}
Oral administration of hydrophobic and poorly intestinal epithelium-permeable drugs is a significant challenge. Herein, we report a new strategy to overcome this problem by using novel, pHresponsive and membrane-active nanogels as drug carriers. Prepared by simple physical crosslinking of amphiphilic pseudopeptidic polymers with pH-controlled membrane-activity, the size and hydrophobicity-hydrophilicity balance of the nanogels could be well tuned. Furthermore, the amphiphilic nanogels could release hydrophobic payloads and destabilise cell membranes at duodenum and jejunum pH 5.0-6.0, which suggests their great potential for intestinal drug delivery.
\end{abstract}

ACS Paragon Plus Environment 
KEYWORDS: Nanogel, pH-responsive, membrane-active, oral delivery, hydrophobic drug

Oral administration is the most widely used drug delivery route, due to its convenience and high patient compliance, especially for the treatment of chronic diseases.1,2 However, the low bioavailability of poorly water-soluble drugs (which account for approximately $40 \%$ of the marketed drugs) remains a critical issue. ${ }^{3}$ Moreover, some of these poorly soluble drugs, e.g., amphotericin B, colistin, ritonavir and paclitaxel, have low membranepermeability in the gastrointestinal (GI) tract, which further limits their efficacy. ${ }^{4}$ Thus, there is urgent need to develop oral delivery systems to enhance both the solubility and the membranepermeability of hydrophobic and poorly intestinal epitheliumpermeable drugs.

Nanogels, defined as nano-sized hydrogels, have attracted considerable attention due to their unique combination of the dimensional advantages of nanotechnology with the hydrophilicity and flexibility of hydrogels. ${ }^{5-7}$ In particular, those nanogels with amphiphilic nature are of great interest due to their capability of encapsulating poorly water-soluble payloads. ${ }^{8-12}$ Recently a series of nanogels composed of cholesterol conjugated polyvinyl alcohol (PVA) have been developed for encapsulation of hydrophobic 
drugs including paclitaxel and a geldanamycin analogue 17-AAG. ${ }^{13}$ The mucus-adhesive properties of PVA along with the large surface area of the nanogels successfully enhanced the adsorption in the GI tract. ${ }^{14}$ However, the vinyl polymers are not biodegradable in vivo, and the bioavailability enhanced by passive intestinal adsorption instead of active absorption is limited.

Herein, we report a novel biodegradable nanogel carrier with pHcontrolled membrane-activity to achieve active intestinal absorption and efficient intestinal release of hydrophobic and poorly intestinal epithelium-permeable drugs. It was prepared by physically crosslinking the pH-responsive, fusogenic, alkyl chaingrafted pseudopeptides, PLP-NDA, previously developed in our group. PLP-NDA18, containing 18 mol\% decylamine (NDA) relative to the carboxylic acids pendant to the poly ( backbone, is one of the comb-like, amphiphilic polymers showing vastly superior membrane activity. ${ }^{15}$ At slightly acidic pH 5.0-6.0 typical of duodenum and jejunum¹6, PLP-NDA18 destabilised cell membranes, thus facilitating payloads translocation. Apart from this pH range, negligible membrane activity was observed. This property could be utilised to precisely control the intestinal cell membrane permeability. In this study, PLP-NDA nanogels exhibited comparable membrane-permeability to the free PLP-NDA polymers, which indicates their potential for enhancing drug delivery through intestinal membrane cells. Nile Red (NR) was 
encapsulated into the PLP-NDA nanogels as a hydrophobic model drug. The controlled release kinetics favoured the simulated intestinal fluids rather than the simulated gastric fluids. The release profile could be further tuned by controlling the hydrophobicityhydrophilicity balance of the PLP-NDA polymer with a different percentage of NDA conjugation. The use of membrane-active polymers in nanogel formation is much easier and more convenient to enhance intestinal drug delivery, compared with surface modifications of drug carriers that usually require lengthy chemical synthesis and purification. Therefore, this strategy provides a new insight into the intestinal drug carrier design and preparation.

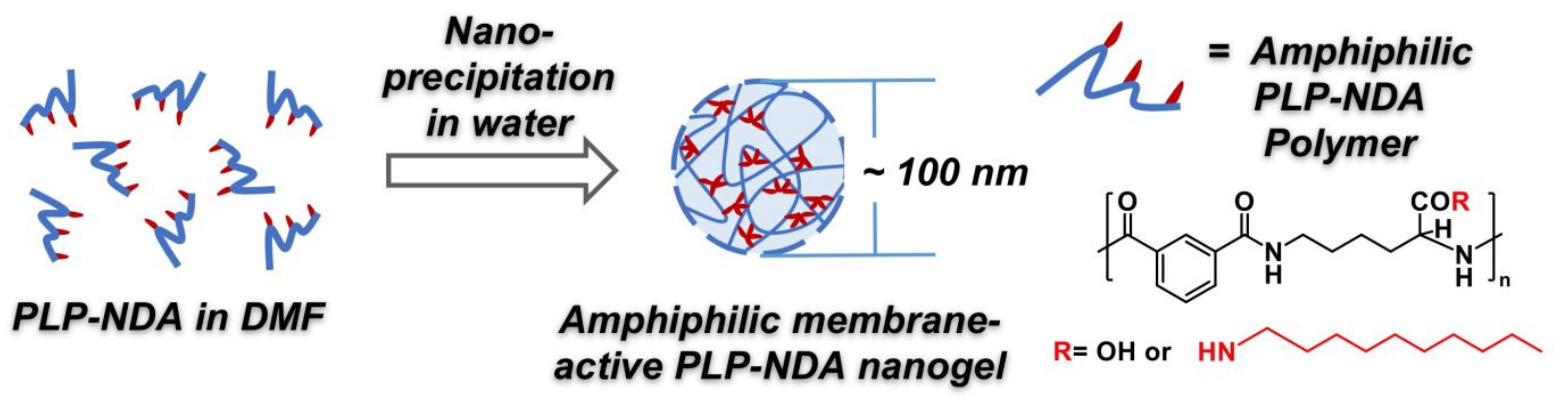

Scheme 1. Synthetic scheme of the amphiphilic, membrane-active, physically crosslinked PLP-NDA nanogel.

As shown in scheme 1, the PLP-NDA nanogel was physically crosslinked via nanoprecipitation of pH-responsive, membraneactive, amphiphilic PLP-NDA polymers in an aqueous solution (details shown in the Supporting Information). Briefly, PLP-NDA polymers were first dissolved in dimethylformamide (DMF) at 
various concentrations, and then the polymer DMF solutions were dropped into deionised water under vigorous stirring. The effects of nanoprecipitation parameters on the PLP-NDA18 nanogel size and polydispersity were investigated systematically. The Z-average sizes of 18 different formulations (details shown in Table s1 in the Supporting Information) are mapped in Figure 1a. The hydrodynamic sizes measured by dynamic light scattering (DLS) were plotted against the initial polymer concentration in DMF and the final polymer concentration in water. The sloping lines refer to different water/DMF ratios. It was found that the nanogel size was almost proportional to the PLP-NDA18 polymer concentration in either solution. A higher polymer concentration (either in DMF or water) generally led to larger particle formation, which is consistent with previous reports about nanoprecitation.17-19 This trend was also confirmed by the DLS particle size distribution shown in Figure Sla and Slb in the Supporting Information, in which the DLS peak shifted to a higher value with increasing the polymer concentration in water and DMF, respectively. However, when the initial polymer concentration in DMF was too high $\left(\geq 100 \mathrm{mg} \mathrm{mL}^{-1}\right.$, marked by red crosses), large aggregate formation was observed by eye and also indicated in the DLS quality report. A high local concentration of PLP-NDA18 in DMF caused polymer chains to overlap and entangle together, which hindered the diffusion of polymers. ${ }^{19}$ When the polymer was too dilute in water $\left(\leq 0.02 \mathrm{mg} \mathrm{mL}^{-1}\right.$, marked by 
blue crosses), it was not favourable for particle formation, leading to insufficient nanoparticles detected by DLS. This concentration threshold for particle formation is in accordance with our previous report of the critical aggregation concentration (CAC) of PLP-NDA18 at $0.031 \mathrm{mg} \mathrm{mL}^{-1} \cdot{ }^{15}$ Only at a polymer concentration higher than the CAC, interactions between the alkyl side chains of PLP-NDA18 became significant and led to selfassembly, which was detected by a hydrophobic probe, pyrene. ${ }^{15}$ Considering all these factors, a grey area was defined in Figure la for PLP-NDA18 nanogel preparation. Within this area, the nanogels with a suitable size and a favourable dispersity (PDI < 0.2) could be synthesised.

(a)

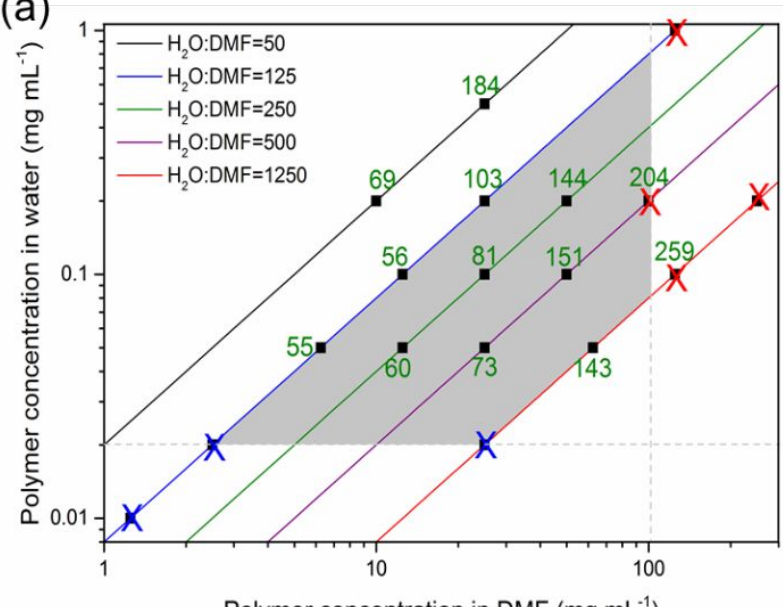

(b)

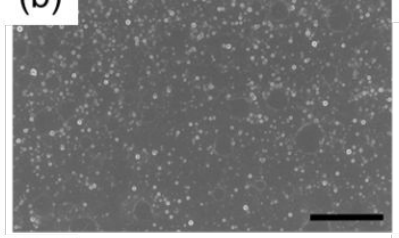

(d)

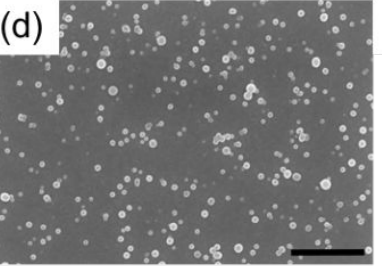

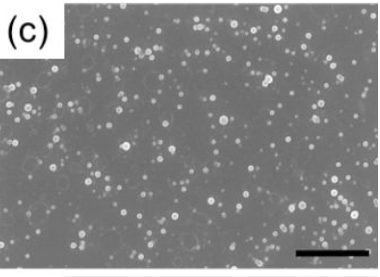

(e)

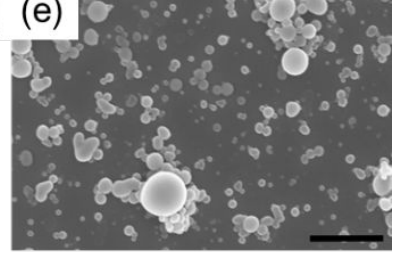

Polymer concentration in DMF $\left(\mathrm{mg} \mathrm{mL}^{-1}\right)$

Figure 1. (a) A 2-D map of hydrodynamic sizes of the PLP-NDA18 nanogels as a function of PLP-NDA18 polymer concentrations in DMF and in water. Green numbers represent the Z-average size (nm) of the corresponding formulation measured by DLS; the blue cross means 
there were not enough particles in the sample for DLS measurement; the red cross means there were large particles in the sample and the data failed to pass the Malvern Zetasizer internal quality test. Each data point was the average of three measurements. (b), (c), (d) and (e), SEM images of representative PLP-NDA18 nanogel formulations, with an average hydrodynamic size of $55.7 \pm 0.7 \mathrm{~nm}$, $103.0 \pm 2.8 \mathrm{~nm}, 151.4 \pm 3.7 \mathrm{~nm}$ and $259.2 \pm 3.3 \mathrm{~nm}$ respectively (according to DLS measurements). The scale bar represents $1 \mu \mathrm{m}$.

The representative PLP-NDA18 nanogels were then visualised by SEM to investigate their morphology. Figure 1 b shows the smallest nanogel (55.7 $\pm 0.7 \mathrm{~nm}$ determined by DLS) obtained in the formulation trials described in Figure la. Most of the particles retained a spherical shape and individually distributed on the substrate. This suggests that the nanogels had good colloidal stability due to the strong hydrophobic interactions between the pendant $\mathrm{C}_{10}$ alkyl side chains of PLP-NDA18. The diameter of the particles shown on the SEM image varied from 30 to $100 \mathrm{~nm}$, which is in good agreement with the DLS results. Figure 1c and 1d represent the median-sized PLP-NDA18 nanogels $(103.0 \pm 2.8 \mathrm{~nm}$, $151.4 \pm 3.7 \mathrm{~nm}$ respectively determined by DLS), which were also well-distributed with spherical shapes, though larger than those in Figure 1b. Figure le shows an example formulation (259.2 \pm 3.3 
nm determined by DLS) red-crossed in Figure 1a. The morphology of these nanogels was significantly different from the other three shown in Figurelb, $1 \mathrm{c}$ and $1 \mathrm{~d}$. Large particle aggregates with irregular shapes were prevalent in Figure le. This is also consistent with the DLS results, in which large particles were reported.

To investigate the hydrophobic drug loading capacity of the amphiphilic PLP-NDA nanogels, a common model cargo, NR, was loaded in situ during the nanogel preparation. The NR loaded in the nanogel had much stronger absorbance at $580 \mathrm{~nm}$ (Figure s2a in the Supporting Information), compared with the same concentration of free NR dispersed in water. This indicates that the hydrophobic NR was encapsulated in the hydrophobic compartments of the amphiphilic nanogels. The FTIR spectra (Figure s2b in the Supporting Information) show that the characteristic peaks of NR were visible in the NR-loaded nanogel, further confirming the successful loading of hydrophobic payloads in the PLP-NDA nanogels. Quantitative results indicate that the loading efficiency (mass percentage of the NR loaded relative to the PLPNDA nanogel used for NR loading) was $16.7 \pm 1.0 \%$ and the encapsulation efficiency (mass percentage of the NR loaded relative to the $\mathrm{NR}$ fed) was $1.7 \pm 0.1$ wto, comparable to the NR loading in the nanogels prepared by other researchers using poly(vinyl alcohol)-b-poly (N-vinylcaprolactam) or oligo(ethylene 
glycol).20,21 After loading, the size of the PLP-NDA18 nanogel was $76.2 \pm 0.8 \mathrm{~nm}$, with a PDI of $0.10 \pm 0.01$. The Zeta potential was $-29.9 \pm 1.2 \mathrm{mV}$. The negative charge was attributed to the deprotonation of the carboxylic acid groups in PLP-NDA18. The particle size and zeta potential of the PLP-NDA18 nanogel were monitored for one month and negligible variations were detected. SEM images measurement showed that the nanogel morphology was also well retained and no particle aggregation was observed during the one-month storage (Figure $\mathrm{S} 2 \mathrm{C}$ in the Supporting Information). Those results further demonstrated their good colloidal stability. The NR release study was performed in bio-relevant buffers including the fasted-state simulated gastric fluid (FaSSGF) and the fasted-state simulated intestinal fluid (FaSSIF). FaSSGF and FaSSIF contain biosurfactants, lecithin and sodium taurocholate, which are bio-derived emulsifying reagents for hydrophobic drug absorption. ${ }^{22}$ Compared with other hydrophobic drug release studies involving the use of artificial surfactants for drug solubilisation, the release buffers used in this study would better mimic the in vivo environment and provide a better understanding of the bioavailability in vivo. 

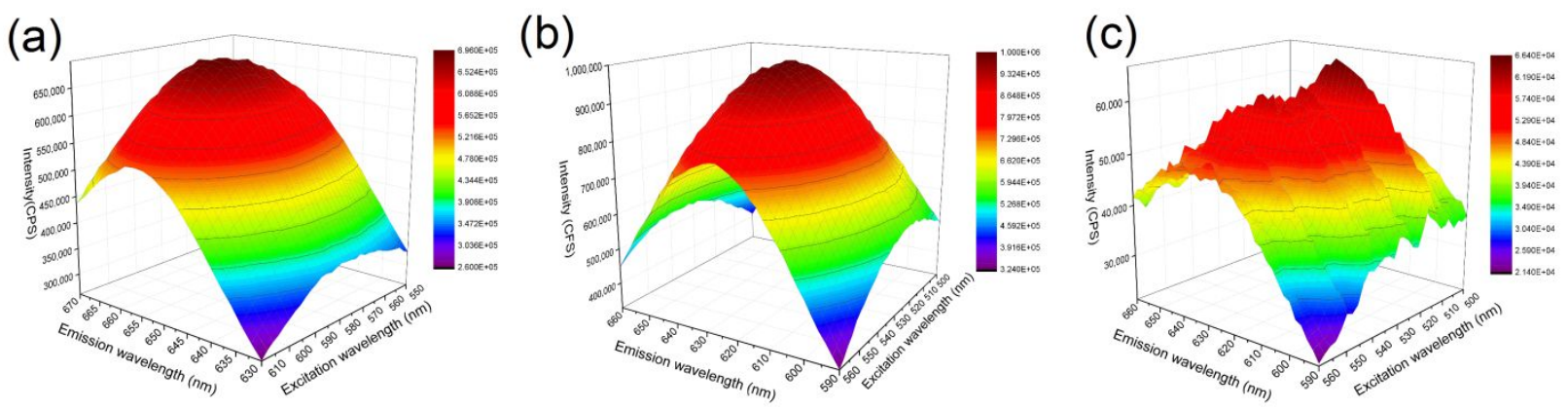

Figure 2. The 3D-fluorescence spectra of the NR loaded in the PLPNDA18 nanogels in (a) deionised water, (b) FaSSIF and (c) FaSSGF. According to the 3D fluorescence spectrometry study shown in Figure 2, NR was released from the PLP-NDA18 nanogel in the simulated GI fluids, and the intestinal environment was much more favourable for NR release than the gastric environment. As previously reported, NR was sensitive to its surrounding environment. ${ }^{23,24}$ When its local environment changes, the fluorescence excitation/emission wavelengths as well as the intensity can vary significantly. As shown in Figure 2a, the NRloaded PLP-NDA18 nanogel sample in water had the excitation and emission peaks at 570 and $660 \mathrm{~nm}$, respectively. After the NR release in FaSSIF, the excitation peak shifted to $520 \mathrm{~nm}$ and the emission peak shifted to $630 \mathrm{~nm}$ (Figure 2b). The significant blue shifts of both peaks indicated the increased hydrophobicity of the surrounding environment of $N R$, 23 which was attributed to emulsification of the released NR in biosurfactants. After the NR release in FaSSGF, similar blue shifts of both excitation (515 nm) and emission peaks $(630 \mathrm{~nm})$ were also observed (Figure 2c). 
However, the intensities of both peaks in FaSSGF were significantly lower than those in FaSSIF. This suggests that NR was only partially released and emulsified by similar biosurfactants in FaSSGF, while most NR still remained encapsulated in the nanogels. The quantitative study of the release kinetics also suggests that the PLP-NDA nanogels favoured the release in FaSSIF rather than in FaSSGF. As shown in Figure 3a, the NR release in FaSSIF was always significantly higher than that in FaSSGF within the period of time studied. Around 60\% of hydrophobic payloads were released from the PLP-NDA18 nanogel in FaSSIF within the first 5 hours. Considering that the average time of human intestinal transition is approximately 3-4 hours, 25,26 the release rate was sufficient for intestinal delivery. As comparison, the NR release from the PLPNDA18 nanogel in FaSSGF was not significant, because only less than 25\% release was observed in the first 3 hours, which is the average gastric transition time. ${ }^{26}$ The much quicker release in FaSSIF was attributed to the pH-dependent change of the PLP-NDA polymer conformation. As previously reported, the polymers displayed a globule-to-coil transition of conformation when $\mathrm{pH}$ increased from 3 to $5 .{ }^{15}$ The transition was caused by the deprotonation of carboxylic acid groups, which led to the enhanced electrostatic repulsion between negatively charged carboxylate ions. As a result, the polymers displayed an expanded structure, weakening the physical crosslinking within the PLP-NDA nanogels. 
Thus, the hydrophobic NR payloads tended to diffuse out rather than retained in the nanogels. Once released, the NR was solublised by the biosurfactants in FaSSIF, which was proved by the results shown in Figure 2 .

The release kinetics could be modulated by simply controlling the hydrophobic domains of the amphiphilic nanogels. The nanogel prepared using the PLP-NDA10 polymer with a lower NDA-conjugation density at 10 mol\% was used for NR loading and release. The NRloaded PLP-NDA10 nanogel had a similar hydrodynamic size (81.3 $0.5 \mathrm{~nm})$ and zeta potential (-26.59 $\pm 0.49 \mathrm{mV})$, but slightly lower loading efficiency (12.3 $\pm 1.3 \%)$ due to the less hydrophobic domains for NR incorporation. In FaSSGF, the 3h-cumulative NR release of the PLP-NDA10 nanogel was similar to the PLP-NDA18 nanogel (Figure 3b). However, in FaSSIF, the release of hydrophobic payloads from the PLP-NDA10 nanogel was much quicker and more efficient. The 5h-cumulative release reached $71.4 \%$, and after $24-$ hour incubation almost complete release was achieved. This suggests that the PLP-NDA10 nanogel with less hydrophobic domains is more suitable for payloads that require quick release with multiple doses, despite a slightly lower loading efficiency. 
(a)

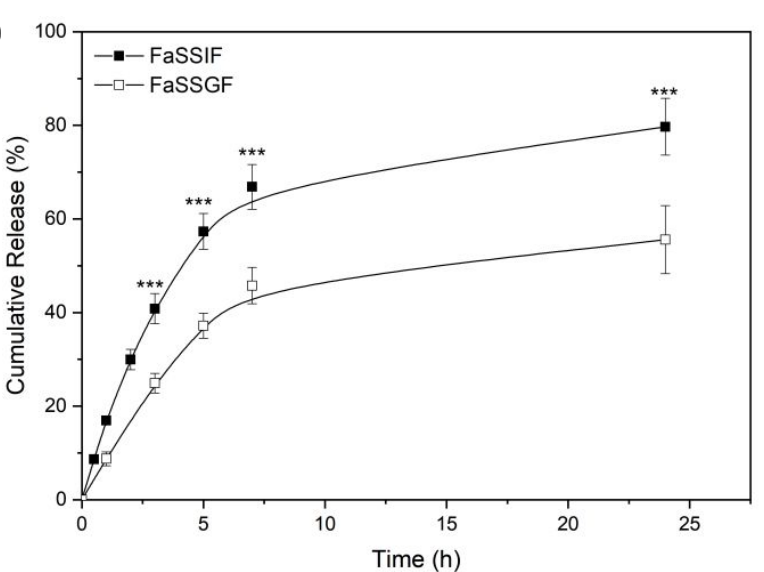

(b)

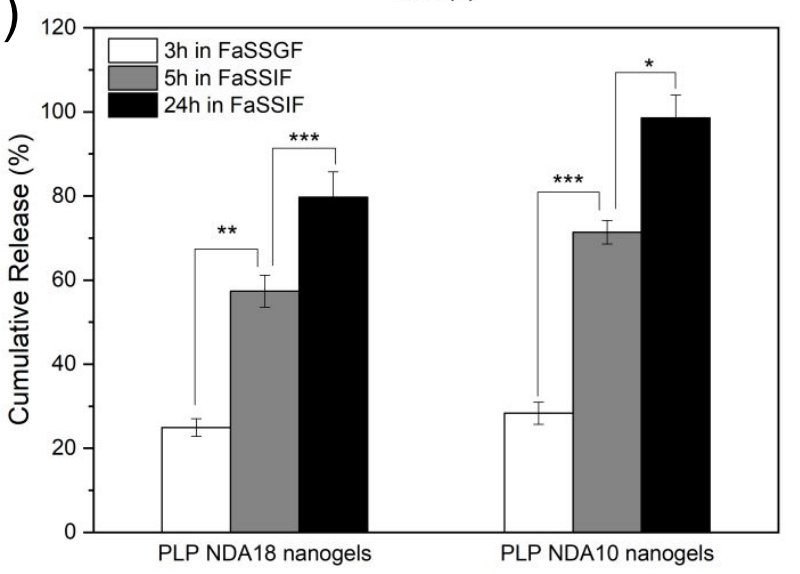

Figure 3. (a) NR release kinetics of the PLP-NDA18 nanogel in FaSSIF and FaSSGF respectively. (b) Comparison of the 3hcumulative NR release in FaSSGF, as well as the $5 \mathrm{~h}-$ and $24 \mathrm{~h}-$ cumulative NR release in FaSSIF between the PLP-NDA10 and PLPNDA18 nanogels. The single asterisk symbol (*), double asterisk symbol (**) and triple asterisk symbol $(* * *)$ denote to $p<0.05, p<0.01$ and $p<0.001$, respectively.

The pH-dependent membrane-lytic activity of the PLP-NDA nanogels was characterised by haemolysis assay. As shown in Figure 4a, the relative haemolysis of the PLP-NDA18 nanogel was comparable to free PLP-NDA18 polymer within the pH range tested. The haemolytic 
activity of both materials was negligible at pH 7.4, but considerably increased when $\mathrm{pH}$ dropped to 5.5. Their relative haemolysis then significantly decreased when pH further decreased to 4.5. The PLP-NDA18 nanogel had almost the same maximum hemolytic activity (65.6\% $\pm 0.1 \%)$ at $\mathrm{pH} 5.5$ with the free PLP-NDA18 polymer (65.5\% $\pm 0.6 \%$ ) at the equivalent polymer concentration. This suggests that the pH-responsive membrane-lytic activity of the PLP-NDA18 polymer was well retained ${ }^{15}$ after physical crosslinking to form the nanogel. Similarly, the haemolytic activity of the PLP-NDA10 nanogel was also comparable to the free PLP-NDA10 polymer, as shown in Figure $\mathrm{S} 3$ in the Supporting Information. The pH-induced membrane activity of the PLP-NDA nanogels within the pH range (5.06.0) typical of duodenum and jejunum ${ }^{16}$ facilitates precise control of the intestinal cell membrane permeability. This, together with the favourable, pH-triggered drug release in the intestinal environment, would improve the bioavailability of poorly soluble drugs through oral administration. 

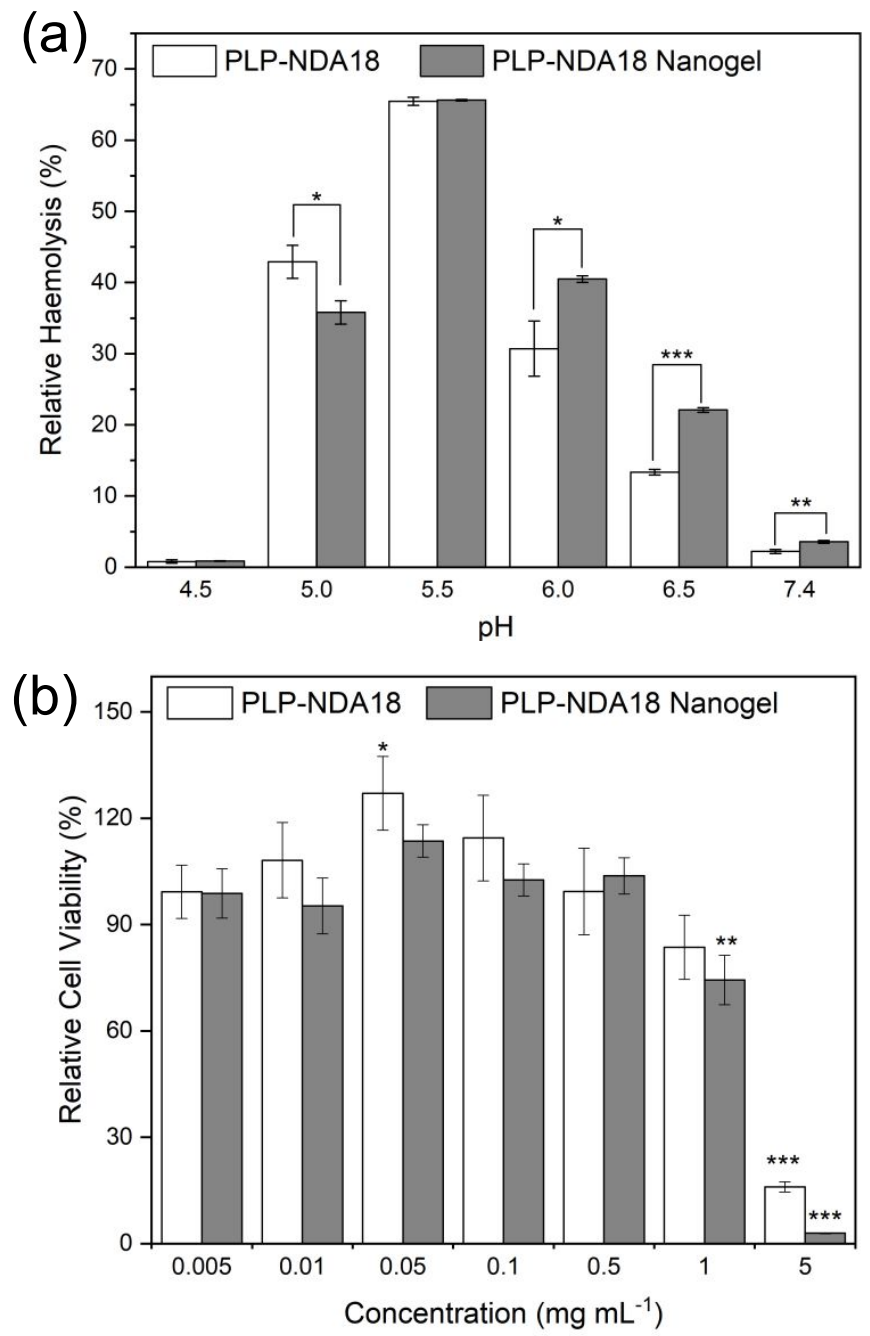

Figure 4. (a) The membrane-lytic activity of the PLP-NDA18 polymer and the physically crosslinked PLP-NDA18 nanogel in buffers at pH ranging from 4.5 to 7.4. The final concentration of both the PLPNDA18 and the PLP-NDA18 nanogel in different buffers was $0.02 \mathrm{mg}$ $\mathrm{mL}^{-1}$. (b) The cytotoxicity of the PLP-NDA18 polymer and the physically crosslinked PLP-NDA18 nanogel towards Hela cells after 24 h of treatment, as evaluated by Alamar Blue assay. The single asterisk symbol $(*)$, double asterisk symbol (**) and triple asterisk symbol $(* * *)$ denote to $p<$ $0.05, p<0.01$ and $p<0.001$, respectively. 
Although PLP-NDA based nanogels demonstrated significant, pHcontrolled membrane activity at a very low concentration $10.02 \mathrm{mg}$ $\mathrm{mL}^{-1}$ ), negligible cytotoxicity towards Hela cells was observed at the same concentration. Actually, as shown in Figure 4b, the PLPNDA18 nanogel displayed a similar cytotoxicity profile to the free PLP-NDA18 polymer, showing no cytotoxic effects in a wide concentration range from 0.005 to $0.5 \mathrm{mg} \mathrm{m}^{-1}$. A similar observation was obtained on the cytotoxicity of the PLP-NDA10 nanogel and the free PLP-NDA10 polymer, as shown in Figure s4 in the supporting Information.

In summary, we have demonstrated a new strategy for the oral delivery of hydrophobic and poorly intestinal epithelium-permeable drugs using the novel, pH-responsive, membrane-active nanogels, which were physically crosslinked by the biomimetic, amphiphilic pseudopeptidic PLP-NDA polymers. Prepared by simple nanoprecipitation method, the size of these amphiphilic PLP-NDA nanogels could be well controlled with a narrow polydispersity. The profiles of loading and release of Nile red as a hydrophobic model drug showed the desirable release in the simulated intestinal fluids rather than the simulated gastric fluids. Moreover, the nanogels enhanced cell membrane permeability considerably at intestinal pH. The nanogels showed no cell cytotoxicity at a

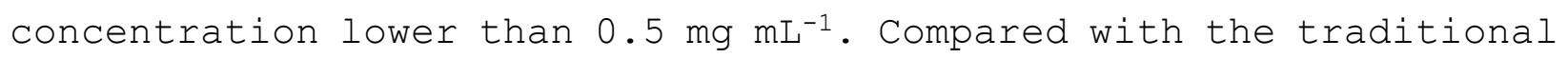
nanogels composed of mucus-adhesive, non-biodegradable polymers 
for passive intestinal adsorption, the novel, amphiphilic PLP-NDA nanogels were prepared by the biodegradable pseudopeptides with pH-controllable membrane activity and could enable the active intestinal absorption. The integrated intestinal cell membrane permeability and intestinal drug release suggest promising potential of the amphiphilic PLP-NDA nanogels in oral delivery of hydrophobic and poorly intestinal epithelium-permeable drugs.

\section{ASSOCIATED CONTENT}

\section{SUPPORTING INFORMATION}

The supporting information is available free of charge on the ACS Publications website at http://pubs.acs.org. Experimental details and supporting data including PLP-NDA18 nanogel formulations and their hydrodynamic sizes measured by DLS; absorbance spectra, FTIR and SEM of the Nile red-loaded PLP-NDA18 nanogels; pH-dependent membrane-lytic activity and cytotoxicity of the PLP-NDA10 polymer and the PLP-NDA10 nanogels.

\section{AUTHOR INFORMATION}

\section{Corresponding Author}

* Tel $:+44$ (0) 20 75942070. Fax: +44 (0) 20 75945638. * E-mail:

rongjun.chen@imperial.ac.uk. 


\title{
Author Contributions
}

R.C. and S.W. designed and supervised the research. S.W., Y.H., X.H., B.C. and W.S. performed the research. S.W. and R.C. prepared the manuscript. All authors have read, commented on, and approved the final version of the paper.

\section{Notes}

The authors declare no competing financial interests.

\section{DATA ACCESS STATEMENT}

Data underlying this article can be accessed on Zenodo (wWw.zenodo.org) at DOI: $10.5281 /$ zenodo.1336886, and is openly available under the Creative Commons CC-BY licence.

\section{ACKNOWLEDGMENTS}

The work was financially supported by the Marie Curie Actions under EU FP7 Initial Training Network SNAL 608184 .

\author{
REFERENCES \\ (1) Tibbitt, M. W.; Dahlman, J. E.; Langer, R. Emerging \\ Frontiers in Drug Delivery. J. Am. Chem. Soc. 2016, 138 (3), 704- \\ 717.
}


(2) Sharpe, L. A.; Daily, A. M.; Horava, S. D.; Peppas, N. A. Therapeutic Applications of Hydrogels in Oral Drug Delivery. Expert Opin. Drug Deliv. 2014, 11 (6), 901-915. Kawabata, Y.; Wada, K.; Nakatani, M.; Yamada, S.; Onoue, S. Formulation Design for Poorly Water-Soluble Drugs Based on Biopharmaceutics Classification System: Basic Approaches and Practical Applications. Int. J. Pharm. 2011, 420 (1), 1-10. Ghadi, R.; Dand, N. BCS Class IV Drugs: Highly Notorious Candidates for Formulation Development. J. Control. Release 2017, $248,71-95$

Ekkelenkamp, A. E.; Elzes, M. R.; Engbersen, J. F. J.; Paulusse, J. M. J. Responsive Crosslinked Polymer Nanogels for Imaging and Therapeutics Delivery. J. Mater. Chem. B 2018, 6, $210-$ 235 .

(6) Liu, G.; An, Z. Frontiers in the Design and Synthesis of Advanced Nanogels for Nanomedicine. Polym. Chem. 2014, 5 (5), 15591565 .

(7) Kabanov, A. V.; Vinogradov, S. V. Nanogels as Pharmaceutical Carriers: Finite Networks of Infinite Capabilities. Angew. Chemie - Int. Ed. 2009, 48 (30), 5418-5429. Lee, W. C.; Li, Y. C.; Chu, I. M. Amphiphilic Poly(D, LLactic Acid)/Poly(Ethylene Glycol)/Poly(D, L-Lactic Acid) Nanogels 
for Controlled Release of Hydrophobic Drugs. Macromol. Biosci. $2006,6(10), 846-854$.

Asadi, H.; Rostamizadeh, K.; Salari, D.; Hamidi, M. Preparation and Characterization of Tri-Block Poly(Lactide)poly(Ethylene Glycol)-poly(Lactide) Nanogels for Controlled Release of Naltrexone. Int. J. Pharm. 2011, 416 (1), 356-364.

(10) Wei, J.; Wang, H.; Zhu, M.; Ding, D.; Li, D.; Yin, Z.; Wang, L.; Yang, Z. Janus Nanogels of PEGylated Taxol and PLGA-PEGPLGA Copolymer for Cancer Therapy. Nanoscale 2013, 5 (20), 9902.

(11) Abandansari, H. S.; Abuali, M.; Nabid, M. R.; Niknejad, H. Enhance Chemotherapy Efficacy and Minimize Anticancer Drug Side Effects by Using Reversibly $\mathrm{PH}-$ and Redox-Responsive Cross-Linked Unimolecular Micelles. Polymer (Guildf). 2017, 116, 16-26.

(12) Wei, X.; Senanayake, T. H.; Bohling, A.; Vinogradov, S. V. Targeted Nanogel Conjugate for Improved Stability and Cellular Permeability of Curcumin: Synthesis, Pharmacokinetics, and Tumor Growth Inhibition. Mol. Pharm. 2014, 11 (9), 3112-3122.

(13) Senanayake, T. H.; Lu, Y.; Bohling, A.; Raja, S.; Band, H.; Vinogradov, S. V. Encapsulation of Poorly Soluble Drugs in Polymer-Drug Conjugates: Effect of Dual-Drug Nanoformulations on Cancer Therapy. Pharm. Res. 2014, 31 (6), 1605-1615. 
(14) Senanayake, T. H.; Warren, G.; Wei, X.; Vinogradov, S. V. Application of Activated Nucleoside Analogs for the Treatment of Drug-Resistant Tumors by Oral Delivery of Nanogel-Drug Conjugates. J. Control. Release 2013, 167 (2), 200-209.

(15) Chen, S.; Wang, S.; Kopytynski, M.; Bachelet, M.; Chen, R. Membrane-Anchoring, Comb-Like Pseudopeptides for Efficient, PHMediated Membrane Destabilization and Intracellular Delivery. ACS Appl. Mater. Interfaces 2017, 9 (9), 8021-8029.

(16) Ovesen, L.; Bendtsen, F.; Tage-Jensen, U.; Pedersen, N. T.; Gram, B. R.; Rune, S. J. Intraluminal PH in the Stomach, Duodenum, and Proximal Jejunum in Normal subjects and Patients with Exocrine Pancreatic Insufficiency. Gastroenterology 1986, 90 (4), $958-962$.

(17) Lepeltier, E.; Bourgaux, C.; Couvreur, P. Nanoprecipitation and the "Ouzo Effect": Application to Drug Delivery Devices. Adv. Drug Deliv. Rev. 2014, 71, 86-97.

(18) Cheng, J.; Teply, B. A.; Sherifi, I.; Sung, J.; Luther, G.; Gu, F. X.; Levy-Nissenbaum, E.; Radovic-Moreno, A. F.; Langer, R.; Farokhzad, O. C. Formulation of Functionalized PLGA-PEG Nanoparticles for in Vivo Targeted Drug Delivery. Biomaterials $2007,28(5), 869-876$. 

Legrand, P.; Lesieur, S.; Bochot, A.; Gref, R.; Raatjes, W.; Barratt, G.; Vauthier, C. Influence of Polymer Behaviour in Organic Solution on the Production of Polylactide Nanoparticles by Nanoprecipitation. Int. J. Pharm. 2007, 344 (1-2), 33-43. Liu, J.; Detrembleur, C.; Hurtgen, M.; Debuigne, A.; De Pauw-Gillet, M.-C.; Mornet, S.; Duguet, E.; Jérôme, C. Reversibly Crosslinked Thermo- and Redox-Responsive Nanogels for Controlled Drug Release. Polym. Chem. 2014, 5 (1), 77-88.

(21) Qiao, Z. Y.; Zhang, R.; Du, F. S.; Liang, D. H.; Li, Z. C. Multi-Responsive Nanogels Containing Motifs of Ortho Ester, Oligo(Ethylene Glycol) and Disulfide Linkage as Carriers of Hydrophobic Anti-Cancer Drugs. J. Control. Release 2011, 152 (1), $57-66$

(22) Holm, R.; Müllertz, A.; Mu, H. Bile Salts and Their Importance for Drug Absorption. Int. J. Pharm. 2013, 453 (1), $44-$ 55.

(23) Greenspan, P.; Fowler, S. D. Spectrofluorometric Studies of the Lipid Probe, Nile Red. J. Lipid Res. 1985, 26 (7), 781789.

(24) Mukherjee, S.; Raghuraman, H.; Chattopadhyay, A. Membrane Localization and Dynamics of Nile Red: Effect of 
Cholesterol. Biochim. Biophys. Acta - Biomembr. 2007, 1768 (1), $59-66$

(25) Borgström, B.; Dahlqvist, A.; Lundh, G.; Sjövall, J. Studies of Intestinal Digestion and Absorption in the Human. $J$. Clin. Invest. 1957, 36 (10), 1521-1536.

(26) Christensen, F. N.; Davis, S. S.; Hardy, J. G.; Taylor, M. J.; Whalley, D. R.; Wilson, C. G. The Use of Gamma Scintigraphy to Follow the Gastrointestinal Transit of Pharmaceutical Formulations. J. Pharm. Pharmacol. 1985, 37 (2), 91-95. 


\section{TABLE OF CONTENTS GRAPHIC}

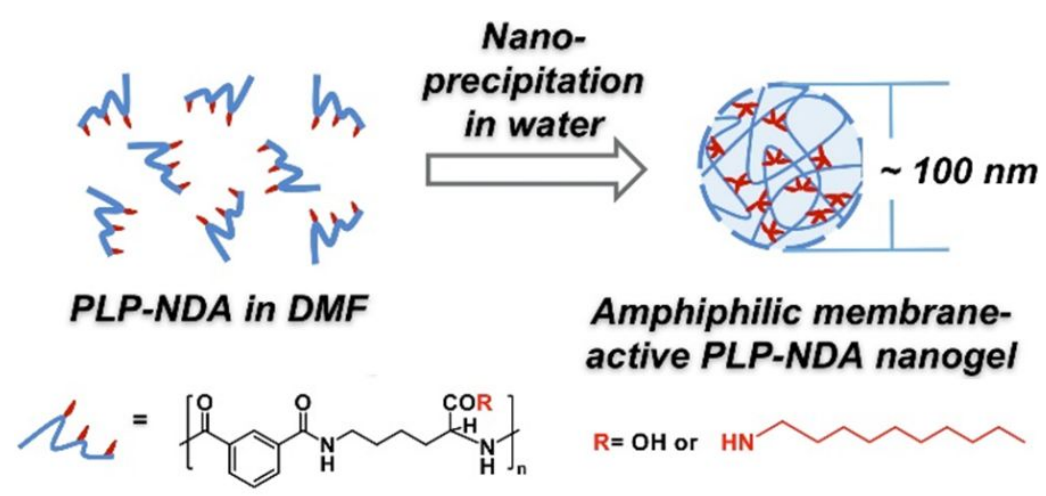

ACS Paragon Plus Environment 